\title{
Articles périodiques 1998
}

\section{Raymonde Monnier}

\section{(2) OpenEdition}

\section{Journals}

\section{Édition électronique}

URL : https://journals.openedition.org/ahrf/1996

DOI : 10.4000/ahrf.1996

ISSN : 1952-403X

Éditeur :

Armand Colin, Société des études robespierristes

Édition imprimée

Date de publication : 1 décembre 1999

Pagination : 791-799

ISSN : 0003-4436

\section{Référence électronique}

Raymonde Monnier, "Articles périodiques 1998 », Annales historiques de la Révolution française [En ligne], 318 | octobre-décembre 1999, mis en ligne le 12 avril 2006, consulté le 23 avril 2022. URL : http://journals.openedition.org/ahrf/1996 ; DOl : https://doi.org/10.4000/ahrf.1996

Ce document a été généré automatiquement le 23 avril 2022.

Tous droits réservés 


\section{Articles périodiques $1998^{1}$}

\section{Raymonde Monnier}

\section{Numéros spéciaux}

1 •Annales Historiques Compiégnoises, n 71-72. Les élections sous la Révolution dans l'Oise et en Picardie, $70 \mathrm{p}$.

2 S. BIANCHI, Voter, élire pendant la Révolution française.

3 C. GUT, Les lois électorales de la Révolution.

4 H. SIMON, Les plébiscites révolutionnaires dans l'Oise (1793, 1795 et 1799).

5 J. BERNET, L'élection de l'évêque et des curés constitutionnels dans l'Oise (1791-1793).

6 L. DRUGEON, Les élections des juges durant la Révolution française (1790-1799): l'exemple du département de l'Oise.

7 M. EDELSTEIN, Participation et sociologie électorales dans l'Aisne en mai 1790 et juin 1791.

8 J. BERNET, Sources et bibliographie sur les élections de la période révolutionnaire dans l'Oise.

9 - Cahiers d'histoire, $n^{\circ}$ 69, 1997. Dossier: Les communistes et le bicentenaire de la Révolution française. Introduction de Roger Martelli.

10 Articles de Serge woliKow, Claude MAZAURIC, Roger BOURDERon, Frédéric genevéE, Alexandre CouRBAN, Présentation d'une bibliographie: la Révolution française et l'édition communiste (1920-1989).

11 - Études d'Histoire du Droit et des Idées politiques, Presses Universitaires des Sciences sociales de Toulouse, 1997, $\mathrm{n}^{\circ}$ 1, $428 \mathrm{p}$. Actes du colloque de Toulouse (1995). Justice et politique.La Terreur dans la Révolution française, s. d. Germain Sicard.

12 •French Historical Studies, vol. 21, n 3, pp. 383-414.Forum : A crisis in History? On Gérard Noiriel's sur la "Crise de l'Histoire », articles de J. W. Scott et L.Kramer.

-Histoire de l'éducation, nos 79-80, Bibliographie d'histoire de l'éducation française, 340 p.

14 •Historia, $\mathrm{n}^{\circ}$ 616, Dossier : Bonaparte en Égypte.

15 M. BATTESTI, Le rêve de posséder la terre des pharaons. 
Ph. MASSON, Des premières victoires à la capitulation.

J. TULARD, Desaix.

M. BATTESTI, Aboukir.

M. CHEHAB, Sans Béchir, pas de conquête de Constantinople.

R. LEBEAU, Les savants en Égypte.

F. LEMAIRE, Desgenettes et Lavrey mobilisés contre la peste.

-Historia, $\mathrm{n}^{\circ}$ 624, Dossier : La Vendée.

Articles de R. SECHER, M. GALLO, A. TEXIER, D. LAMBERT.

-International Review for social history, vol. suppl. $\mathrm{n}^{\circ}$ 6, New Methods for Social History, edited by Larry J. Griffin and Marcel van der Linden, $165 \mathrm{p}$.

-L'Histoire, n² 216, décembre 1997. Dossier : La découverte des Pyramides.

P. BRET, L'expédition d'Égypte : une révolution scientifique.

M. DEWACHTER, Champollion en Orient.

P. BRET, Vrais et faux mystères des Pyramides.

H. LAURENS, Bonaparte a-t-il colonisé l'Égypte?

G. ANDREU, Vivant Denon, bienfaiteur du Louvre.

-Revue d'Histoire Littéraire de la France, $\mathrm{n}^{\circ}$ 6. Chateaubriand, $336 \mathrm{p}$.

Articles de A. MICHEL, M. DELON, J.-P. CLÉMENT, E. TABET, J.-C.CAVALLIN, F. BERCEGoL, T. WEBERMAILLOT, S. MENANT.

-Politeia, $\mathrm{n}^{\circ}$ 1-2, Centre de Philosophie Politique de l'Université de Reims. Simon Nicolas Henri Linguet (1736-1794). Politique, philosophie, économie et leur critique au XVIII siècle, 227 p.

D. GAY LEVY, Linguet et la création d'une sphère publique oppositionnelle.

A. TALAZAC-LAURENT, Simon Linguet critique du système judiciaire.

F. MARKOVITS, Linguet et les images du droit dans la Théorie des lois civiles.

M. MEURISSE, Religion et société : Linguet et le divorce.

GAROUX, Le fanatisme des philosophes et l'art d'écrire de Simon Linguet.

M.C. ROYER, Linguet, Condorcet et le despotisme.

F. RICHI, Un rêve de Linguet : le laboureur du roi.

F. GAUTHIER, La guerre du blé au XVIII ${ }^{e}$ siècle. Linguet critique des économistes.

H. GRANGE, La loi d'airain des salaires dans la pensée de Linguet et de Necker.

D. BARUCH, Linguet, du pamphlet au polar.

G. NAVET, De l'autre côté du Tibre (Marx et Linguet).

Articles 1998

ABAD R., Les tueries à Paris sous l'Ancien Régime ou pourquoi la ville n'a pas eu d'abattoirs aux XVII ${ }^{\mathrm{e}}$ et XVIII ${ }^{\mathrm{e}}$ siècles, Histoire, Économie, Société, $\mathrm{n}^{\circ} 4$.

ALIMENTO A., Un gioco di scacchi? Aspetti e problemi di storia politica della Francia pre-rivoluzionaria, Rivista Storica Italiana, $\mathrm{n}^{\circ}$ 1, pp. 529-572.

4 ARCHES P., Guerre de Vendée et sources démographiques pendant la Révolution et l'Empire. Essai critique sur les Deux-Sèvres, Recherches vendéennes, 1997, $\mathrm{n}^{\circ} 4$, pp. 101-146. 


\section{Paris, de} Neptunia, $\mathrm{n}^{\circ} 209$. l'Histoire, $\mathrm{n}^{\circ} 1$. 127-137.

AYMES J.R., Napoléon et Joseph Bonaparte sous le regard des Espagnols, Clefs pour

BAILEY C.R., Municipal Government and Secondary Education During the Early French Revolution : Did Decentralization Work? French History, pp. 25-42.

BATTESTI M., Aspects navals de l'expédition d'Égypte, Revue Historique des Armées, $\mathrm{n}^{\circ} 2$.

BEAUREPAIRE, P.-Y., Un creuset maçonnique dans l'Europe des Lumières : la loge de la Candeur, Orient de Strasbourg, Revue d'Alsace, t. 124, fasc. 602, pp. 89-120.

-, Le cosmopolitisme des Lumières à l'épreuve : la Réunion des étrangers à l'Orient de BELISSA, Marc, La guerre de course dans les débats parlementaires (1792-1795),

-, Droit des gens et constitutionnalisme dans la pensée des Lumières, Revue historique de droit français et étranger, $\mathrm{n}^{\circ}$ d'avril-juin.

BERNEDE A., Sur les rives du Nil, juillet 1798, la tactique ou l'art d'appliquer le règlement, Revue de la Société des Amis du Musée de l'Armée, n¹16.

BERNET J., Attichy [Oise], de l'Ancien Régime à l'époque contemporaine: données administratives, économiques et sociales. - Attichy et son canton sous le Directoire (1795-1799), Annales Historiques Compiégnoises, n 73-74, pp. 5-29.

-, L'élection de l'évêque et des curés constitutionnels dans l'Oise sous la Révolution, Revue du Nord, t. LXXX, nos 326-327, pp. 637-656.

BEUGNES Dr., Givet [Ardennes] durant la période révolutionnaire (1789-1800), Ardenne wallonne, 1997, nos 68 à 71 .

9 BIARD M., Agents de centralisation ou médiateurs politiques? Entre intendants et préfets, l'expérience des représentants en mission (1793-1795), Les Limites de siècles, lieux de ruptures novatrices depuis les temps modernes, Annales Littéraires, Université de Franche-Comté, vol.668, pp. 363-374.

BIDOUZE F., Discours parlementaire et culture politique. Le parlement de Navarre, DixHuitième Siècle, $\mathrm{n}^{\circ}$ 30, pp. 347-359.

BIRCHALL I. H., Babeuf and the oppression of women, The British Journal for EighteenthCentury Studies, 1997, vol. 20, n 1, pp. 63-75.

BLACK J., Rewriting Revolution, European History Quarterly, 1997, vol. 27, n 1, pp.

BOIS P. A., 1789-1806-1815 : quel XIX siècle pour l'Allemagne? Les Limites de siècles, lieux de ruptures novatrices depuis les temps modernes, Annales Littéraires, Université de Franche-Comté, vol. 668, pp. 427-440.

BOUR I., Sensibilité et répétition dans les romans révolutionnaires de Mary Hays, Études Anglaises, vol. 51, n², pp. 143-155.

BRANCA S., voir GUILHAUMOU J.

BREEN T.H., HALL T., Structuring Provincial Imagination : The Rhetoric and Experience of Social Change in Eighteenth-Century New-England, The American Historical Review, $\mathrm{n}^{\circ}$ 5, pp. 1411-1439. 

l'Ancien Régime, Revue Historique des Armées, $\mathrm{n}^{\circ} 4$. $\mathrm{n}^{\circ} 4$, pp. 746-774. architectural particulier, Histoire, Économie, Société, $\mathrm{n}^{\circ} 4$. Storica Italiana, 1997, $\mathrm{n}^{\circ}$ 3, pp. 803-852. $\mathrm{XVIII}^{\mathrm{e}}$ siècle), Revue d'Histoire Moderne et Contemporaine, $\mathrm{n}^{\circ} 2, \mathrm{pp} .323-345$. Women, French Historical Studies, vol. 21-1, pp. 181-192. Storica Italiana, $\mathrm{n}^{\circ} 2$, pp. 573-641. Century Life, 1997, vol. 21, n² 2, pp. 203-221. vol. 43/1, pp. 1-29. 49, $\mathrm{n}^{\circ}$ 6, pp. 1573-1599. Storica Italiana, 1997, $\mathrm{n}^{\circ}$ 3, pp.938-960. post-Robespierrista, Società e Storia, 1997, a. 20, n 76, pp.377-400. (1789-1801), Langue et langage, $\mathrm{n}^{\circ}$ 7, pp. 97-121. Dix-Huitiémiste. Jean Mainil (ed.), Nottingham French Studies, Spring, 37, n²1, pp. 17-25. et du Béarn, $\mathrm{n}^{\circ} 25$, pp. 205-223. Antoine Jullien de Paris, Rivista Storica Italiana, n², pp. 411-462.

BROUILLET P., L'organisation de la Maréchaussée dans la généralité de Paris à la fin de

CAILLY C., Structure sociale et consommation dans le monde proto-industriel rural textile : le cas du Perche Ornais au XVIII ${ }^{\mathrm{e}}$ siècle, Revue d'Histoire Moderne et Contemporaine,

CARBONNIER Y., Les maisons à ponts parisiens fin XVIIIe siècle: un phénomène

CERETTI M., Alexandro Verri : lettere sulla rivoluzione francese (1791-1800), Rivista

CHABAUD G., Images de la ville et pratiques du livre : le genre des guides de Paris (XVII ${ }^{\mathrm{e}}$

COLE J.R., Debunking Roussel's « Report » on the Society of Revolutionary Republican

CUAZ M., L'identità ambigua : l'idea di «nazione » tra storiografia e politica, Rivista

DE BAECQUE A., Robespierre, monstre-cadavre du discours thermidorien, Eighteenth

DECEULAER H., Guildsmen, Entrepreneurs, and Market Segments: the Case of the Garment Trades in Anvers and Gand $\left.\left(\mathrm{XVI}^{\mathrm{e}}-\mathrm{XVIII}\right)^{\mathrm{e}}\right)$, International Review for social history,

DEFALVARD H., Valeur et contrats à la lumière de Turgot [1769], Revue économique, vol.

7 DE FRANCESCO A., Per una rilettura della cultura politica del Risorgimento. Giacobinismo e moderatismo nella biografia di Gioacchino Mario Olivier-Poli, Rivista

78 -, Dalla Montagna a Bonaparte : la carriera politica di Jean-Pierre Audouin nella Francia

DELEPLACE M., Un exemple d'approche lexicologique en histoire : la notion d'anarchie

DELON M., Les nouveaux objets de la recherche dix-huitiémiste en France, La Recherche

81 -, Luxe et luxure. Réflexions à partir de Sade, French Erotic Fiction. Ideologies of Desire,

82 -, Du danger de la littérature, Le corps sadien, Europe, $n^{\circ}$ 835-836, pp. 3-8 et pp. 22-33.

3 DE ROSA L., Immobility and Change in Public Finance in the Kingdom of Naples, 1649-1806, The Journal of European Economic History, vol. 27, n 1, pp. 9-28.

DESAN S., « War between Brothers and Sisters »: Inheritance Law and Gender Politics in Revolutionary France, French Historical Studies, vol.20, 4, 1997, pp. 597-634.

DESPLAT C., Les cahiers de doléances des communautés de Béarn en 1789, Revue de Pau

DI RIENZO E., Nascita di una nazione. La missione in Vandea e in Bretagna di Marc- 
DONATO M.P., La République romaine de 1798-1799. Panorama des études récentes, Revue d'Histoire Moderne et Contemporaine, $\mathrm{n}^{\circ}$ 1, pp.134-140.

D'SOUZA F., Observations de Legoux de Flaix sur l'Inde de la fin du xviII ${ }^{\mathrm{e}}$ siècle, Journal Asiatique, Société Asiatique, t. 286, n 1, pp. 323-358.

DUPORT A.-M., Les mouvements populaires dans les diocèses civils de Nîmes, Uzès et Alès de 1740 à 1789, Annales du Midi, n² 225, pp. 31-45.

DUPUIS P., L'histoire des représentations et la représentation de l'Histoire, Cahiers d'histoire, $\mathrm{n}^{\circ}$ 73, pp. 113-117.

DWYER P. G., War and resistance in napoleonic Europe : some recent works, European History Quarterly, 1997, vol. 27, n 4, pp. 549-562.

ESTÈVE C., Les transformations de la chasse en France : l'exemple de la Révolution, Revue d'Histoire Moderne et Contemporaine, $\mathrm{n}^{\circ}$ 2, pp. 404-424.

FANGET J.-P., Action antiféodale et mouvements paysans en Basse-Auvergne, époque révolutionnaire : 1789-an II, Siècles, 1996, n 4, pp.29-60.

FAVIER R., Lumières et politiques dans les petites villes dauphinoises à la fin du XVIIIe siècle, Cahiers d'Histoire, t. XLIII, nº 3-4, pp. 513-522.

FOGLEMAN A.S., From Slaves, Convicts, and Servants to Free Passengers: The Transformation of Immigration in the Era of the American Revolution, The Journal of American History, $\mathrm{n}^{\circ}$ 1, pp. 43-76.

FOLLAIN A., Les communautés rurales en Normandie sous l'Ancien Régime. Identité communautaire, institutions du gouvernement local et solidarités, Revue d'Histoire Moderne et Contemporaine, $\mathrm{n}^{\circ}$ 1, pp. 691-721.

GAIN P., Ambiguïtés et leçon d'une liturgie laïque : la commémoration républicaine de la mort de Louis XVI dans quelques villes et villages du Nord de l'an V à l'an VII, Revue du Nord, t. LXXX, nos 326-327, pp. 681-696.

GALASSO G., Rivoluzione francese e lettere italiane : la prima grande opera di Paul Hazard, Rivista Storica Italiana, $\mathrm{n}^{\circ}$ 1, pp. 293-302.

GARNOT B., La violence et ses limites dans la France du xVIII siècle: l'exemple bourguignon, Revue Historique, $\mathrm{n}^{\circ}$ 606, pp. 237-253.

GARRIGUES F., Les intendants du commerce au XVIII siècle, Revue d'Histoire Moderne et Contemporaine, $\mathrm{n}^{\circ} 3$, pp. 626-661.

GASPARD C., L'interdisciplinarité à l'épreuve des almanachs de la fin du siècle, DixHuitième Siècle, $\mathrm{n}^{\circ}$ 30, pp. 165-178.

2 GILLI M., La sécularisation de la notion de patriotisme en Allemagne à la fin du XVIII siècle et au début du XIX ${ }^{e}$ siècle, Les Limites de siècles, lieux de ruptures novatrices depuis les temps modernes, Annales Littéraires, Université de Franche-Comté, vol. 668, pp. 387-402.

3 GREEN D.R., Lines of Conflict: Labour Disputes in London, 1790-1870, International Review for social history, vol. 43/2, pp. 203-233.

GRIMMER C., Espace urbain et hiérarchie sociale à Aurillac [XviII ${ }^{e}$ siècle], Cahiers $d u$ Centre d'histoire des entreprises et des communautés (Clermont-Ferrand II), ${ }^{\circ} 7$, pp. 33-42.

GRUDER V.R., Political News as Coded Messages : The Parisian and Provincial Press in the Pre-Revolution. 1787-1788, French History, vol.12/1, pp. 1-24. 
GUILHAUMOU J., Un débat franco-américain autour de la Révolution française, DixHuitième Siècle, $\mathrm{n}^{\circ}$ 30, pp. 245-256.

-, Le tout de la Nation, Portée et limites du discours d'Assemblée (1789-1790), Le Mot : analyse du discours et sciences sociales, Langue et langage, $\mathrm{n}^{\circ} 7$, pp. 123-147.

- et S. BRANCA, De "société " à " socialisme » (Sieyès) : l'invention néologique et son contexte discursif. Essai de colinguisme appliqué, Langage \& Société, n 83-84, pp. 39-77.

GUITTON E., Poétesses et Égéries de 1770 à 1830, Cahiers Roucher-André Chénier, n 17, Textes réunis par Edouard Guitton, $160 \mathrm{p}$.

HAFID-MARTIN N., Évolution et critique de la théorie des climats à travers le XvIII siècle en France. Du déterminisme géographique à la liberté politique in I Notebook dello SWIF, Sito Web Italiano per la Filosofia, http ://lgxserver.uniba.it/lei/swif.htm, an I, $\mathrm{n}^{\circ} 2$, février.

HALL T., voir BREEN T.H.

HANSON P., Monarchist Clubs and the Pamphlet Debate over Political Legitimacy in the Early Years of the French Revolution, French Historical Studies, vol. 21-2, pp. 299-324.

HATCHER J., Labour, Leisure and Economic Thought before the Nineteeth Century, Past and Present, $\mathrm{n}^{\circ}$ 160, pp. 64-115.

4 HAZAREESINGH S., From Democratic Advocate to Monarchist Critic of the Republic: The Penitent Jacobinism of Etienne Vacherot (1809-1897), The English Historical Review, $\mathrm{n}^{\circ} 454$, pp. 1143-1179.

5 HEITZ H., Les années révolutionnaires à Saverne [Bas-Rhin], 1789-1799- 4e partie : la municipalité de Saverne sous l'administration du maire Arth (nov. 1791- févr. 1793), Société d'histoire et d'archéologie de Saverne, 1997, cah. 179, pp. 9-20.

6 HUTA C., Jean Senebier (1742-1809) : Un dialogue entre l'ombre et la lumière. L'art d'observer à la fin du xvIII ${ }^{\mathrm{e}}$ siècle, Revue d'histoire des sciences, 51/1, pp. 93-105.

7 HUUSSEN Jr. A. H., Gezinscriminaliteit in Groningen, Stad en Land, 1750-1810 / Criminalité de famille à Groningue, 1750-1810/, Het Noorden in het midden. Opstellen [...] aangeboden aan F. J. Bakker, D.E.H. de Boer, R.I.A. Nip, R.W.M. van Schaïk red., Assen, Groningse Historische Reeks, 17, pp. 139-166.

118 -, Catherine the Great's Instruction (Nakaz) to her Legislative Commission : the Dutch Translations of 1769 and 1794, Russia and the Low Countries in the Eighteenth Century, E. Waegemans (ed.), Groningue, Baltic Studies, 5, pp. 245-260.

119 JAUME L., Unité et pluralité : la souveraineté révolutionnaire et son héritage, The Tocqueville Review - La Revue Tocqueville, vol. XIX, n² 2, Toronto U.P., pp. 65-85.

120 KING P., The Rise of Juvenile Delinquency in England 1780-1840 : Changing Patterns of Perception and Prosecution, Past and Present, ${ }^{\circ} 160$, pp. 116-166.

121 KLEINERT A., Die deutschsprachigen physikalischen Wörterbücher des 18. Jahrhunderts. Johann Samuel Traugott Gehler und Johann Carl Fischer, Das Achtzehnte Jahrhundert, 22, pp. 129-138.

KLEPP S.E., Revolutionary Bodies: Women and the Fertility Transition in the MidAlantic Region. 1760-1820, The Journal of American History, n³, pp. 910-945. 
KNOPPER F., Les changements de perception chez les voyageurs allemands à la fin du $\mathrm{XVIII}^{\mathrm{e}}$ siècle, Les Limites de siècles, lieux de ruptures novatrices depuis les temps modernes, Annales Littéraires, Université de Franche-Comté, vol. 668, pp. 603-616.

KOMLOS J., The New World's Contribution to Food Consumption during the Industrial Revolution, The Journal of European Economic History, vol. 27, n 1, pp. 67-82.

KOZUL M., Le voyage sadien en Italie : la Révolution française comme politique libertine dans l'Histoire de Juliette, Eighteenth-Century Fiction, vol. 10, n 4, July, pp. 466-481.

KREBS R., Lenz Beitrag zur Werther-Debatte /La contribution de Lenz à la querelle du Werther/, Die deutsche Aufklärung in Spiegel der neueren französischen Aufklärungsforschung /Les Lumières allemandes vues par la recherche française actuelle sur les Lumières/, Aufklärung, 10, 1, pp. 67-79.

KURY L., Les instructions de voyage dans les expéditions scientifiques françaises (1750-1830), Revue d'histoire des sciences, 51/1, pp. 65-91.

KWASS M., A Kingdom of Taxpayers : State, Formation, Privilege and Political Culture in Eighteenth-Century France, The Journal of Modern History, vol. 70, n² 2, pp. 295-339.

LAHAYE P., La défense de la patrie et les volontaires du district de Bourbon-Lancy [Saône-et-Loire] (1791-1793), Échos du Passé Gueugnon, 1997, n 80, pp. 23-32 ; n 81, pp. 30-41.

LANDI S., Censure et culture politique en Italie au XVIII ${ }^{e}$ siècle. Le cas du Grand-Duché de Toscane, Revue d'Histoire Moderne et Contemporaine, $\mathrm{n}^{\circ}$ 1, pp. 117-133.

LANGLOIS C., La fin des guerres de religion : la disparition de la violence religieuse en France au XIX ${ }^{\mathrm{e}}$ siècle, French Historical Studies, vol. 21-1, pp. 3-25.

LE BOZEC C., Élites-Révolution-Transition (1760-1830), Cahiers d'histoire, n 73, pp. 35-45.

LEMAY E.H., Révolution française et consensus au tournant du XVIII ${ }^{e}$ au XIX siècle, Les Limites de siècles, lieux de ruptures novatrices depuis les temps modernes, Annales Littéraires, Université de Franche-Comté, vol. 668, pp. 337-348.

LÉTÉ L., La vente des biens nationaux [dans la Marne], Champagne Généalogie, 1997, n 76, pp. 232-236.

LUCKETT T., Hunting for Spies and Whores : a Parisian riot on the eve of the French Revolution, Past and Present, 156, 1997, pp. 116-143.

LUXARDO H., Bara, Viala, la fabrication d'un mythe révolutionnaire, Clefs pour l'Histoire, $\mathrm{n}^{\circ} 1$.

MANNUCCI E.J., «Rivale des teatro di Atene». I rivoluzionari francesi e l'arte drammatica, Rivista Storica Italiana, $\mathrm{n}^{\circ}$ 1, pp. 251-283.

MARAMBAUD P., Lequinio : la Vendée et le rejet de la Terreur, Recherches vendéennes, 1997, n 4, pp. 305-329.

MARSHALL T., The Constitutional Purpose of the Bill of Rights, The Review of Politics (U.S.A.), vol. 60, nº 1, Winter, pp. 171-177.

MARTIN J.-C., Femmes et guerre civile, l'exemple de la Vendée, 1793-1796, Clio. Histoire, Femmes et Sociétés, 1997, n 5, pp. 97-115.

MATTONE A., SANNA P., La « rivoluzione delle idee » : la riforma delle due università sarde et la circolazione della cultura europea (1764-1790), Rivista Storica Italiana, $\mathrm{n}^{\circ} 3$, pp. 834-942. 

Université de Franche-Comté, vol. 668, pp. 321-336. of Independance, The Journal of American History, $\mathrm{n}^{\circ}$ 2, pp. 411-438. Revolutionary France, Past and Present, n ${ }^{\circ}$ 159, pp.77-112. 11, 1997, pp. 47-64. Metz, Metz, pp. 9-27. Franche-Comté, vol. 668, pp. 617-624. 1997-1998, pp. 153-174. Studies, 31, 1997-1998, pp. 175-197. Littéraires, Université de Franche-Comté, vol. 668, pp. 349-362. français et étranger, 1997, a. 75, n 1, pp. 93-107. pp. 111-209. 137-202. 97-104. 347-367. Entreprises et Histoire, $\mathrm{n}^{\circ} 18, \mathrm{pp} .73-94$. Sciences Sociales, $\mathrm{n}^{\circ}$ 6, pp. 1075-1088. vol. 668, pp. 417-426.

MAUVIEL M., L'anthropologie française naissante à la fin du XVIII ${ }^{\mathrm{e}}$ siècle, Les Limites de siècles, lieux de ruptures novatrices depuis les temps modernes, Annales Littéraires,

McGLONE R.E., Deciphery Memory : John Adams and the Authorship of the Declaration

McMAHON D.M., The Counter-Enlightenment and the Low-Life of Litterature in Pre-

MERCIER-FAIVRE A.-M., Le langage d'images de Court de Gébelin, Politica Hermetica, ${ }^{\circ}$

MICHAUX G., Bibliothèques messines du XVIII siècle, Mémoires de l'Académie Nationale de

MIETH G., La fin du XVIII ${ }^{e}$ siècle-fin des Lumières pour l'Allemagne, Les Limites de siècles, lieux de ruptures novatrices depuis les temps modernes, Annales Littéraires, Université de

MIRZOEFF N., Revolution, Representation, Equality : Gender, Genre, and Emulation in the Académie Royale de Peinture et Sculpture, 1785-1793, Eighteenth-Century Studies, 31,

MITCHELL J. E., Picturing sisters : 1790 Portraits by J.-L. David, Eighteenth-Century

MONNIER R., La rupture révolutionnaire de 1789 et les origines du républicanisme radical, Les Limites de siècles, lieux de ruptures novatrices depuis les temps modernes, Annales

MORABITO M., L'an III et l'héritage du Comité de Salut public, Revue historique de droit

MORICEAU J.-M., La terre et les paysans en France et en Grande-Bretagne aux XVII et $\mathrm{xVIII}^{\mathrm{e}}$ siècles. Un parcours bibliographique critique (1), Histoire et Sociétés rurales, $\mathrm{n}^{\circ} 9$,

MORINEAU M., Malthus : There and Back. From the Period Preceding the Black Death to the Industrial Revolution, The Journal of European Economic History, vol. $27 \mathrm{n}^{\circ} 1$, pp.

MORTIER R., Le néo-classicisme entre sublime et sensibilité, Cahiers de l'AIEF, n 50, pp.

NORCI CAGIANO DE AZEVEDO L., Parigi «nouvelle Rome ", Rome " deuxième capitale de l'empire » nelle concezione di Vivant Denon, Studi romani, 1997, a. 45, nos 3-4, pp.

OKAMBA E., Hommes et industrie sucrière aux Antilles de l'Ancien Régime à nos jours,

OZOUF M., L'idée républicaine et l'interprétation du passé national, Annales Histoire,

PASETSKY G., Salzbourg au tournant entre le XVIII ${ }^{e}$ et le XIX ${ }^{e}$ siècle : un archévêché entre l'Aufklärung, La Révolution et la sécularisation, Les Limites de siècles, lieux de ruptures novatrices depuis les temps modernes, Annales Littéraires, Université de Franche-Comté, 

sociaux entre le $\mathrm{XVIII}^{\mathrm{e}}$ et le $\mathrm{xIX}^{\mathrm{e}}$ siècle, Les Limites de siècles, lieux de ruptures novatrices depuis les temps modernes, Annales Littéraires, Université de Franche-Comté, vol. 668, pp. 293-300.

PÉLAQUIER É., Les mouvements antifiscaux en Languedoc d'après les archives de la Cour des Comptes, Aides et Finances de Montpellier (1660-1789), Annales du Midi, $\mathrm{n}^{\circ} 225$, pp. 5-29.

PETITEAU N., Les vétérans du Premier Empire : un groupe socio-professionnel oublié, Cahiers d'Histoire, t. XLIII, $\mathrm{n}^{\circ} 1$, pp. 25-46.

PLAGNOL-DIEVAL M.-E., Madame de Genlis et la poésie, Poétesses et égéries poétiques de 1770 à 1820, Actes du Colloque international de la Société Roucher-André Chénier (29-30 mars 1996), Cahiers Roucher-Chénier, n 17, pp. 71-90.

PODVIN J.-L., L'expédition de Bonaparte en Égypte. Un nouveau bicentenaire, Historiens Géographes, n 361, pp. 65-72.

POMPONI F., Triennio sardo? Débats sur la Sardaigne à l'époque révolutionnaire, Revue d'Histoire Moderne et Contemporaine, $\mathrm{n}^{\circ}$ 1, pp. 141-146.

PORRET M., Crimes et châtiments : l'œil du médecin-légiste, Dix-Huitième Siècle, $\mathrm{n}^{\circ}$ 30, pp. 37-50.

PY G., Les Livres I à IV des Confessions, Information littéraire, Belles Lettres, avril 1998.

QUINTILI P., Libertà e determinazione naturale nella filosofia medica della Rivoluzione francese. Bordeu, Diderot, Cabanis, Montag, Rome, Fahrenheit 451, n 3, 1998.

RAXON P., Fins de siècles et jeux de miroir dans le regard catholique belge sur la Révolution française, Les Limites de siècles, lieux de ruptures novatrices depuis les temps modernes, Annales Littéraires, Université de Franche-Comté, vol. 668, pp. 349-362.

REGENT F., Presse parisienne et expédition d'Égypte, Revue Historique des Armées, n ${ }^{\circ} 2$.

REGOURD F., La Société royale d'Agriculture de Paris face à l'espace colonial (1761-1793), Bulletin du Centre d'Histoire des espaces atlantiques, pp. 155-194.

REINALTER H., La notion de "Sattelzeit ", révélatrice des changements politiques et RENAUT M.-H., Vagabondage et mendicité. Délits périmés, réalités quotidiennes, Revue Historique, $\mathrm{n}^{\circ} 606$, pp. 287-322.

REYNARD P. C., Manufacturing strategies in the Eighteeth Century : Subcontracting for Growth among Papermakers in the Auvergne, The Journal of Economic History, vol. 58/1, pp. 155-182.

RICHARD Th., La logistique du corps expéditionnaire en Égypte, Revue Historique des Armées, $\mathrm{n}^{\circ} 2$.

RICUPERATI G., Pour une histoire des Lumières d'un point de vue cosmopolite, DixHuitième Siècle, $\mathrm{n}^{\circ}$ 30, pp. 127-142.

RIEUCAU J.-N., « Les entreprises où les hommes s'exposent à une perte dans la vue d'un profit ». Condorcet et l'héritage de D’Alembert, Revue économique, vol. 49, $\mathrm{n}^{\circ}$ 5, pp. 1365-1405.

ROSSET F., Année flamboyante pour Benjamin Constant, Écriture, Lausanne, 52, automne, pp. 257-262.

RUSSELL G., Burke's dagger : theatricality politics and print culture in the 1790s, The British Journal for Eighteenth-Century Studies, 1997, vol. 20, n 1, pp. 1-16. 
SANNA P., voir MATTONE A.

SCHERR A., The limits of republican ideology : James Monroe in Thermidorian Paris. 1794-1795, Mid-America, 1997, vol. 79, n 1, pp. 5-46.

SGARD J., Diderot vu par les Nouvelles ecclésiastiques, Recherches sur Diderot et sur l'Encyclopédie, n² 25, octobre, pp. 9-19.

SONENSCHER M., Enlightenment and Revolution. Review Article, The Journal of Modern History, vol. 70, n 2, pp. 371-383.

-, The Nation's Debt and the Birth of Modern Republic: the French Fiscal Deficit and the Politics of the Revolution of 1789 (Part II), History of Political Thought, 18, 1997, pp. 267-325.

SPEAR F. A., Bibliographie de Diderot, supplément n 7, Diderot Studies, XXVII, pp. 181-222.

STEDMAN JONES G., Une autre histoire sociale? [note critique sur l'historiographie française et anglaise], Annales Histoire, Sciences Sociales, $n^{\circ}$ 2, pp. 383-394.

STEINER Ph., The Structure of Say's economic writings, History of Economic Thought, vol. 5, n' 2, pp. 227-249.

STEWART L., A Meaning for Machines: Modernity, Utility, and Eighteenth-Century British Public, The Journal of Modern History, vol. 70, n 2, pp.259-294.

SULLIVAN C. R., The First Chair of Political Economy in France: Alexandre Vandermonde and the Principles of Sir James Steuart at the Ecole normale of the Year III, French Historical Studies, vol. 20, 4, 1997, pp. 635-664.

SWEET R., Freemen and Independance in English Borough Politicis c.1770-1830, Past and Present, $\mathrm{n}^{\circ}$ 161, pp. 84-115.

TABAKI A., Identité et diversité culturelle : le mouvement des traductions dans le SudEst de l'Europe (xvIII ${ }^{\mathrm{e}}$ siècle-début du XIX) ), Synkrisi/ Comparaison, fasc. 9, pp. 71-91.

TARIN R., L'Esclavage des Noirs, ou la mauvaise conscience d'Olympe de Gouges, DixHuitième Siècle, $\mathrm{n}^{\circ}$ 30, pp. 373-382.

THILLAY A., L'économie du bas faubourg Saint-Antoine, Histoire, économie, Société, nº 4.

UMBACH M., Visual Culture, Scientific Images and German Small-State Politics in the Late Enlightenment, Past and Present, $n^{\circ} 158$, pp. 110-145.

VANDEPLAS B., La grande peur en Haute-Auvergne, Gavroche, ${ }^{\circ} 102$.

VARRY D., Pour de nouvelles approches des archives de la Société typographique de Neuchâtel, The Darnton Debate. Books and Revolution in the Eighteenth Century, Haydn T. Mason (Ed.), Studies on Voltaire and the Eighteenth Century, n' 359, pp. 235-349.

-, Une bibliothèque arrageoise sous la Révolution : les livres de Boucquel de Beauval, Eulalie. Médiathèques, librairies et lecteurs en Nord-Pas-de-Calais, nº 1, pp. 199-211.

VERGA M., Le XVIII ${ }^{\text {e }}$ siècle en Italie: le "Settecento " réformateur? Revue d'Histoire Moderne et Contemporaine, $\mathrm{n}^{\circ}$ 1, pp. 89-116.

VIOLA P., Rivoluzione francese, giacobinismo, legittimazione violenta e communitaria, Quaderni Storici, 1997, a. 22, n 95, pp. 539-556.

VOLPILHAC-AUGER C., De marbre ou de papier? L'histoire ancienne, du XVIII ${ }^{e}$ au XIX siècle, Cahiers de l'Association internationale des études françaises, mai, $\mathrm{n}^{\circ}$ 50, pp. 105-120. 


\section{NOTES}

1.Les articles parus tardivement, qui n'avaient pu être recensés dans la précédente chronique (A.H.R.F., $\mathrm{n}^{\circ}$ 315, pp. 203-212) sont signalés avec leur date. Je remercie les auteurs qui m'ont envoyé des informations ou des tirés à part pour compléter cette chronique. 